\title{
Dermoscopy Use in Primary Care: A Scoping Review
}

\author{
Jonathan A. Fee ${ }^{1}$, Finbar P. McGrady ${ }^{1}$, Cliff Rosendahl2,3, Nigel D. Hart ${ }^{1}$
}

1 Centre for Medical Education, Queen's University Belfast, UK

2 School of Medicine, The University of Queensland, Brisbane, Australia, Brisbane, Australia

3 School of Medicine, Tehran University of Medical Sciences, Iran

Key words: dermoscopy, general practice, primary health care, melanoma, cancer

Citation: Fee JA, McGrady FP, Rosendahl C, Hart ND. Dermoscopy use in primary care: a scoping review. Dermatol Pract Concept. 2019;9(2):98-104. DOI: https://doi.org/10.5826/dpc.0902a04

Accepted: October 15, 2018; Published: April 30, 2019

Copyright: $\odot 2019$ Fee et al. This is an open-access article distributed under the terms of the Creative Commons Attribution License, which permits unrestricted use, distribution, and reproduction in any medium, provided the original author and source are credited.

Funding: Dr. Fee's study fees and maintenance come from HSC R\&D Division, Public Health Agency's GP Academic Research Training Scheme and EAT/5336/17.

Competing interests: The authors have no conflicts of interest to disclose.

Authorship: All authors have contributed significantly to this publication.

Corresponding author: Dr. Jonathan A. Fee, Centre for Medical Education, Queen’s University, Belfast, UK. Email: jfee03@qub.ac.uk

\footnotetext{
ABSTRACT Background: Patients in many countries with new or changing skin lesions will first consult a primary care physician, often called a general practitioner (GP). With the dramatic rise in melanoma incidence over recent decades, dermoscopy offers a tool with an evidence base supporting its use in skin lesion assessment. How GPs use dermoscopy is unclear.

Objectives: A scoping literature review was carried out to examine the current state of published evidence about dermoscopy use in primary care.

Methods: The methodological steps taken in this review followed those developed by Arksey and O'Malley, as revised by Levac and colleagues. Four electronic databases were searched for evidence published up to January 2018 describing the use of dermoscopy in a generalist primary care setting. Seven articles were identified for analysis.

Results: All included articles have been published since 2007. Most were questionnaire studies and revealed that generally a small minority of GPs use dermoscopy, although some jurisdictions such as Australia report greater use. Dermoscopy is generally used only for the assessment of pigmented skin lesions, but is not used consistently. Several perceived barriers to dermoscopy use, including the need for training, have been reported.

Conclusions: There is a paucity of data on dermoscopy use among GPs, and diversity in questionnaire items prevents comparison between jurisdictions. Perceived barriers to dermoscopy use require more in-depth exploration, potentially including qualitative data, to evaluate them more fully. Understanding these factors, including how GPs train in dermoscopy, will be crucial in widening dermoscopy use in primary care.
} 


\section{Introduction}

For the majority of patients in many countries with a new or changing skin lesion, their first consultation with a health care professional will be with a primary care physician, often called a general practitioner (GP). Dermoscopy has been shown to be an effective tool for the detection of melanoma in primary care [1]. Dermoscopy in primary care is a relatively new tool, and little is known about how GPs use dermoscopy. Given the role of dermoscopy in the early detection of melanoma and other skin cancers, understanding how it is used in the primary care setting, as well as identifying what is not yet clear, is important in directing future research and in helping to expand its use.

Scoping literature reviews, usually called scoping reviews, have become an increasingly common approach to providing a descriptive outline of evidence from published literature [2]. They are useful particularly when the aim is to map an area of research for its main concepts, sources, and types of evidence [3]. In the relatively unexplored area of dermoscopy use in a primary care setting, a scoping review was undertaken to examine the extent of research in this area and to identify where gaps in the existing literature appear.

\section{Methods}

Methodological approaches to conducting scoping reviews have been published in the literature. Arksey and O'Malley developed a framework for scoping reviews, and this was refined by Levac and colleagues [3,4]. This was the framework followed in this review.

\section{Research Team}

The research team comprised a general practice specialty trainee (J.A.F.) and 3 GPs (F.P.M., C.R., and N.D.H.) involved in clinical teaching and medical education research.

\section{Research Ethics}

No ethical approval was required for this work, as this was a secondary analysis of published literature within the public domain.

\section{Step 1: Identifying the Research Question}

While the use of dermoscopy has become more commonplace among doctors who specialize in skin cancer care, such as dermatologists, the aim of this review was to investigate the use of dermoscopy in primary care. For this reason an open and inclusive question was formed: What can be known from the literature about how dermoscopy is used in general practice?

\section{Step 2: Identifying Relevant Studies}

Initial informal literature searches were carried out to identify the various terms used in the literature for dermoscopy and
Table 1. Embase Search Terms

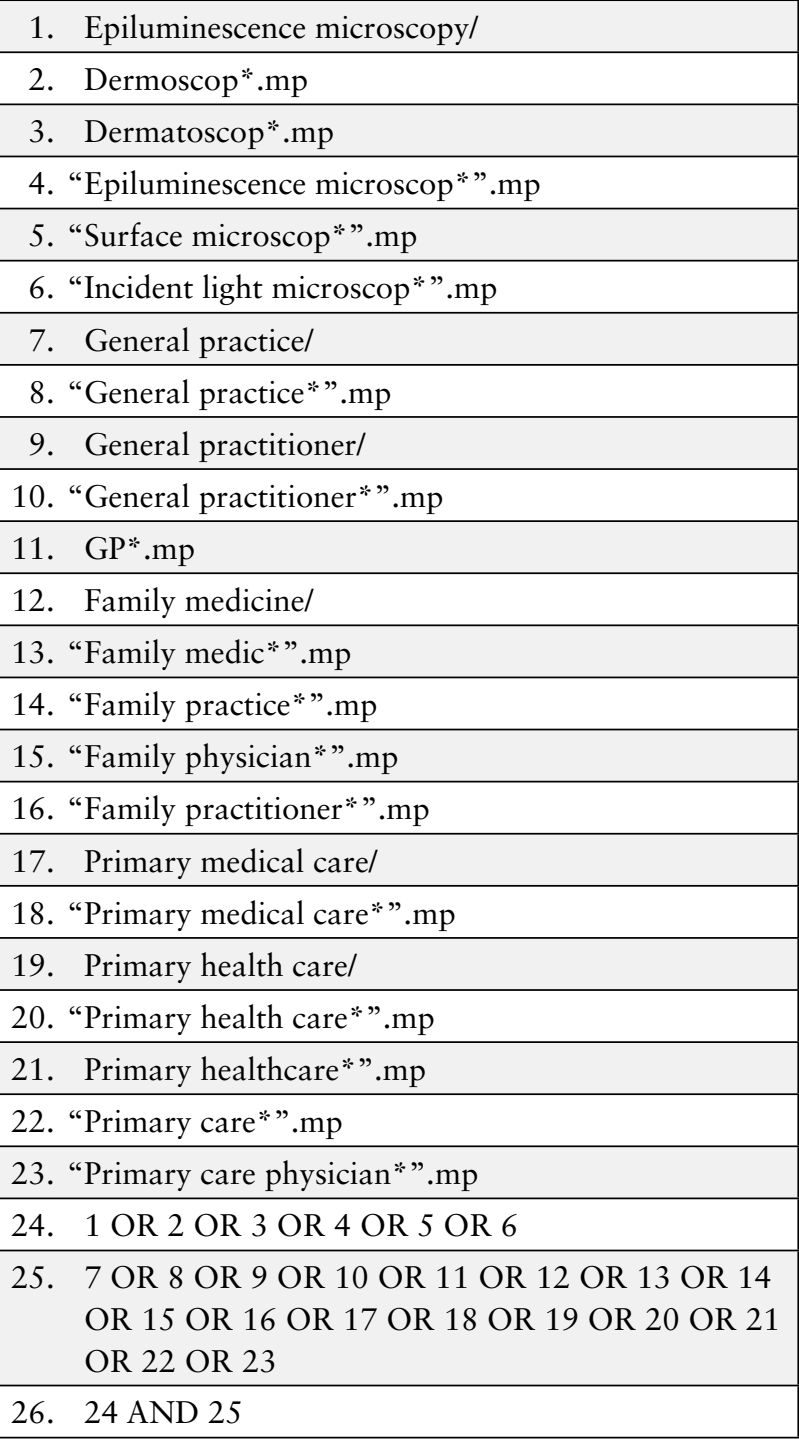

GPs. The expertise of a medical librarian was sought to ensure that there was adequate coverage of relevant databases for formal literature searches.

Formal literature searches were carried out between January and February 2018. Four electronic databases were searched: Embase, MEDLINE, Scopus, and Web of Science. Minor adaptations in search terms were made between databases to account for the different database subject headings. The Embase search strategy is shown in Table 1.

\section{Step 3: Study Selection}

J.A.F. screened abstracts from citations identified in database searches. Where this was insufficient to make a decision, the whole article was read. In the event of ambiguity the article was referred for full-text assessment for eligibility. Articles available only in the form of conference abstracts were excluded at this point, as is standard in scoping review methods. Articles written in languages other than English were also excluded. 
Table 2. Inclusion and Exclusion Criteria for Article Selection

\begin{tabular}{|l|}
\hline Inclusion criteria \\
\hline - Studies examined some aspect of \\
the use of dermoscopy in everyday \\
primary care practice. \\
- Participants were mainly GPs (or in \\
countries where the term GP is not in \\
common use, primary care physicians \\
working in a generalist community \\
setting to whom patients self-refer). \\
\hline Exclusion criteria \\
\hline - Expert reviews of dermoscopy, \\
commentaries or editorials discussing \\
other articles. \\
- Articles focused on GPs working in a \\
specialist or secondary care setting. \\
- GPs recruited to training \\
interventional studies in dermoscopy. \\
- GPs participating in screening \\
programs. \\
- Teledermoscopy studies in which \\
dermatologists interpret the images. \\
Dermoscopy interpreted by artificial \\
intelligence.
\end{tabular}

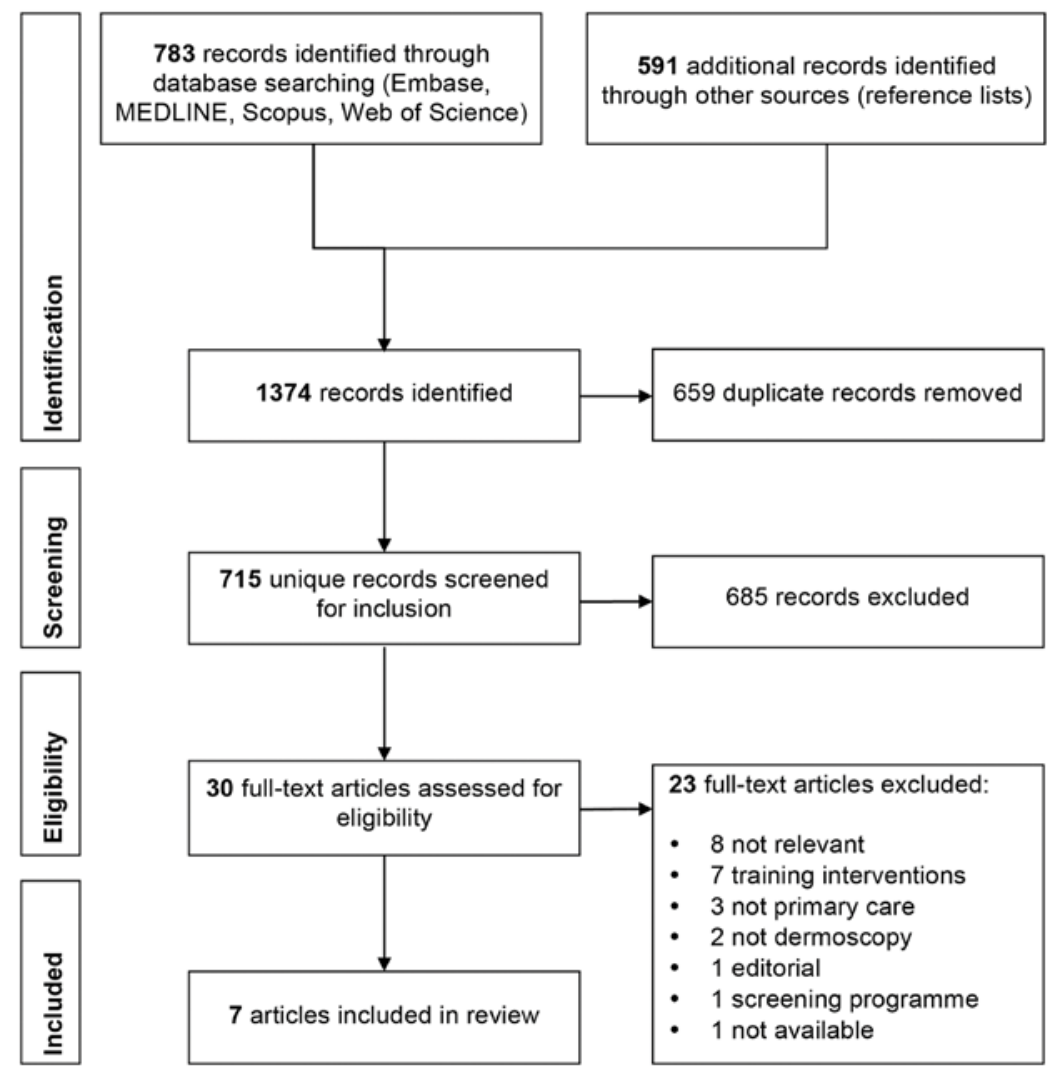

Figure 1. PRISMA flowchart of study selection process [5].
At this stage J.A.F. and N.D.H. met to discuss the articles. Full-text articles were read by both researchers and were considered for inclusion according to the criteria set out in Table 2. Discussion resolved any discrepancies in opinion between the researchers, and consensus was reached.

Whether included in the final analysis or not, the reference lists of all articles reaching this stage were searched, and additional new citations were screened by J.A.F. Articles accepted from reference lists for full-text assessment also had their reference lists searched in an iterative process, until no additional new citations were generated that passed the screening stage.

\section{Step 4: Charting the Data}

J.A.F. created a data extraction sheet using Microsoft Excel (Microsoft, Redmond, WA, USA) and extracted onto it details from the included papers. Data extracted from each article included authors, year of publication, origin of the research, study design, outcome measures, and key findings that related to the review question.

\section{Step 5: Collating, Summarizing, and Reporting the Results}

Preferred Reporting Items for Systematic Reviews and MetaAnalyses (PRISMA) guidance [5] and guidance published by members of the Joanna Briggs Institute and Joanna Briggs
Collaboration Centres was used in reporting the results [6]. A flowchart of the study selection process is shown in Figure 1.

\section{Results}

We identified 783 citations from the database searches. Database searches were carried out sequentially. The search of the fourth database (Embase), after the exclusion of conference abstracts, produced only 4 new citations, none of which passed the screening stage, and database searches were deemed to be sufficient. A large number of additional records were identified through searching reference lists. However, very few were relevant to the review question. Seven articles were included in the review analysis, all of which were observational studies. Of these, 5 were questionnaire studies and 2 reviewed medical records. All had GPs as their subjects. One study included some GPs who did not have generalist practices but were dedicated to skin cancer care; however, as the majority of the participants in the study $(73.6 \%)$ were GPs who maintained generalist practices (albeit some of them had a special interest in skin cancer), this study was included in the review [7].

\section{Origins of Research}

Articles describe work that has been carried out in 4 different countries, as is shown in Figure 2. Australia, the Netherlands, 
and the United States have contributed 2 articles each to the field. All of the research has been conducted in countries with high-income and advanced economies [8,9], and with very high human development in the Human Development Index [10].

\section{Year of Publication}

The first article included in the review was published in 2007. Figure 3 shows a cumulative frequency chart of publication dates. All included articles were published over a 10-year period, and the majority were published in 2016 or 2017.

\section{Use of Dermoscopy by GPs}

Five questionnaire studies and 2 records-based observational studies have described dermoscopy use in primary care. The results are summarized in Table 3.

Dermoscopy use varies widely between jurisdictions. Dermoscopy use is well established in Australia, where between $34 \%$ and $42 \%$ of GPs in generalist practices reported using dermoscopy and where research into the use of dermoscopy in primary care has been undertaken since 2007 [7,11]. High levels of dermoscopy use were also

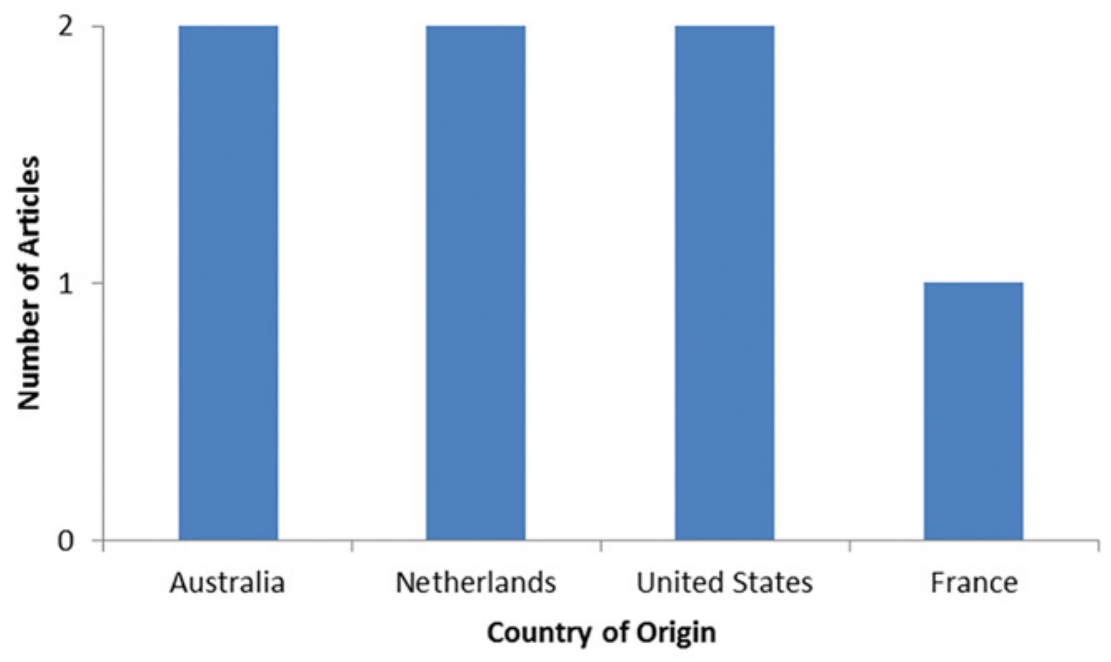

Figure 2. Origins of review articles.

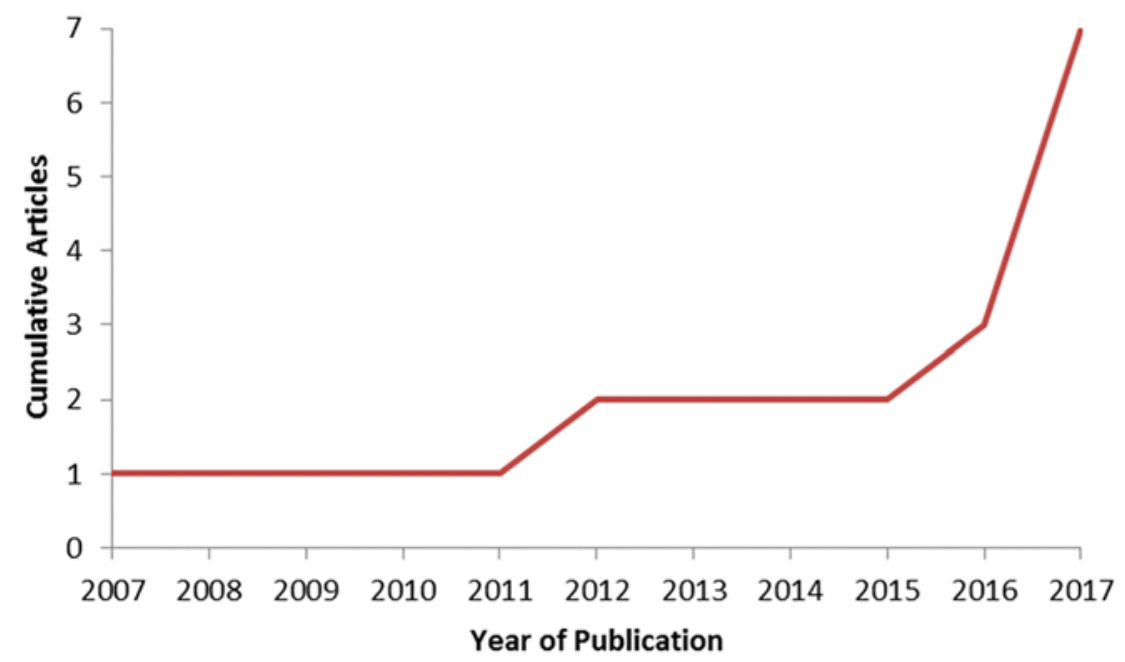

Figure 3. Year of publication of review articles by cumulative frequency.

Table 3. Studies Reporting Use of Dermoscopy by GPs

\begin{tabular}{|c|c|c|c|c|c|}
\hline Study & Year & Country & $\begin{array}{c}\text { No. of } \\
\text { Participants }\end{array}$ & $\begin{array}{l}\text { Response } \\
\text { Rate }\end{array}$ & Use of Dermoscopy \\
\hline Morris et al [15] & 2017 & USA & 768 GPs & Not reported & $\begin{array}{l}6 \% \text { currently use; } \\
15 \% \text { have ever used dermoscopy }\end{array}$ \\
\hline Morris et al [16] & 2017 & USA & 705 GPs & Not reported & $\begin{array}{l}8.3 \% \text { currently use; } \\
19.5 \% \text { have ever used dermoscopy }\end{array}$ \\
\hline Secker et al [12] & 2017 & Netherlands & 309 GPs & Not reported & $37 \%$ use dermoscopy \\
\hline Ahmadi et al [14] & 2017 & Netherlands & $\begin{array}{l}11 \text { GPs; } 580 \\
\text { consultations }\end{array}$ & Not applicable & $8.4 \%$ of consultations use dermoscopy \\
\hline Chappuis et al [13] & 2016 & France & 425 GPs & $10.5 \%$ & $8 \%$ have access to a dermatoscope \\
\hline Rosendahl et al [7] & 2012 & Australia & 193 GPs & Not applicable & $\begin{array}{l}42.6 \% \text { of generalist GPs use; } 89.4 \% \\
\text { of GPs with skin cancer special } \\
\text { interest use; and } 95.9 \% \text { of GPs } \\
\text { dedicated to skin cancer care use } \\
\text { dermoscopy at least weekly }\end{array}$ \\
\hline Chamberlain et al [11] & 2007 & Australia & 223 GPs & $>90 \%$ & $34 \%$ ever use dermoscopy \\
\hline
\end{tabular}


reported among GPs in the Netherlands [12]. In contrast to this, dermoscopy use seems to be lower among GPs in the United States and France, at 6\%-8\%.

One study found that the use of dermoscopy by GPs increases as they either develop a special interest in skin cancer or enter a practice dedicated to skin cancer care. However, when other variables such as practice type were controlled for, no association was found between dermoscopy use and the number of suspicious lesions excised per melanoma diagnosis [7]. However, the authors acknowledge that it was difficult to study dermoscopy use and subspecialization into skin cancer care in isolation, as the two are highly correlated.

How studies reported dermoscopy use varied. Current and previous use of dermoscopy by GPs, GPs' access to a dermatoscope, and consultations that have used dermoscopy were all variably described. One study reported participants' use of dermoscopy as low, medium, or high, with "low" including those who used dermoscopy less than once a week or not at all [7]. One questionnaire simply reported whether participants had ever or never used dermoscopy [11]. These differences prevent clear comparisons between studies and jurisdictions.

\section{Patterns of Dermoscopy Use}

Two studies reported specific information about frequency of dermoscopy use and for what purpose dermoscopy is used in clinical practice. In one study, $20 \%$ of GPs working in generalist practices reported using dermoscopy for all pigmented lesions and $22 \%$ reported using it most days [7]. Another study reported that of $8 \%$ of GPs with access to a dermatoscope, $52 \%$ used it more than once a week [13]. This suggests that GPs who use dermoscopy are not using it consistently; this was highlighted in another study, which found that dermoscopy was used in only $8.4 \%$ of consultations involving skin lesions suspected of malignancy [14].

One study suggested that dermoscopy seems to be used mostly for consultations involving pigmented skin lesions: $82 \%$ of dermoscopy users reported using dermoscopy for the assessment of pigmented skin lesions, $68 \%$ for nonpigmented lesions, and $8 \%$ to allow for digital data transmission, such as teledermoscopy [13].

\section{Characteristics of Dermoscopy Users}

Three studies subanalyzed their questionnaire data to explore whether certain groups of GPs are more likely to use dermoscopy. Results were conflicting. One study found that male GPs were more likely to use dermoscopy than female GPs [13], although other studies did not support this finding $[15,16]$. Two of the studies found that older GPs were more likely to use dermoscopy $[13,16]$, while another reported that younger GPs were more likely to be using the tool [15]. One study reported that GPs in busier practices, who saw more than 400 patients per month, were more likely to use dermoscopy [16], although another study by the same authors did not confirm this [15]. One study demonstrated that GPs subspecializing in skin cancer care were more likely to use dermoscopy than GPs working in generalist GP practices [7].

\section{Perceptions of Dermoscopy Use}

Three questionnaire studies asked participants to rank their perceptions of barriers to dermoscopy use from a list of suggestions. A pattern emerged across the studies that revealed common perceived barriers. The costs of dermoscopy-both the equipment cost and the insufficient reimbursement for its use in practice-were a clear barrier to its use. The other most commonly cited barriers were the need for dermoscopy training and the time needed both for training and to use dermoscopy in practice $[13,15,16]$.

One of the studies also asked participants to rank their perceptions of the advantages of dermoscopy from a list of suggestions. The most common responses were that it helped to diagnose melanoma earlier, that it helped to reduce dermatology referrals, and that it reduced patients' anxiety [13].

Two questionnaire studies asked about confidence. Confidence was significantly higher among dermoscopy users than nonusers in both studies: for the analysis of pigmented skin lesions generally in one study [13] and for differentiating between cancerous and noncancerous skin lesions in the other [15]. One study asked about how dermoscopy affects clinical decision-making; $75 \%$ of dermoscopy users felt that it influenced their diagnoses in practice [11].

\section{Discussion}

\section{Principal Findings}

This review identified 7 observational studies that have investigated the use of dermoscopy in general practice, mostly by means of questionnaires. The use of dermoscopy by GPs varies between jurisdictions; however, because of the paucity and heterogeneity of data it is difficult to draw any firm conclusions. In general, it is a small minority of GPs who use dermoscopy, and only where GPs have subspecialized in skin cancer care do a majority use dermoscopy [7]. Those GPs who use dermoscopy do not do so consistently for all pigmented skin lesions [7], and most use it only for the assessment of pigmented skin lesions [13]. This raises a concern that the dermoscopic features of skin cancers such as amelanotic melanomas may be overlooked.

GPs who used dermoscopy reported feeling more confident in analyzing pigmented skin lesions [13] and in differentiating between cancerous and noncancerous skin lesions [15]. What is unclear is whether dermoscopy makes GPs feel more confident, or whether GPs with more confidence in dermatology are more likely to embrace a new tool for 
skin lesion assessment. Insights such as these are not readily uncovered in questionnaire studies.

GPs perceive the barriers to dermoscopy use to include the cost of the equipment, the need for training, and the time required for training and using dermoscopy in practice. GPs perceived the advantages of dermoscopy to be the earlier diagnosis of melanoma, reducing dermatology referrals, and reducing patient anxiety.

\section{Limitations}

Although this review was carried out according to a recognized scoping review methodology, it was exploratory in nature. Databases and reference lists were searched using a comprehensive set of search terms; however, it is impossible to guarantee that other papers of relevance were not overlooked. As only published articles are included in search databases, any unpublished data, or data that exist in the form of conference abstracts, were not included in the review. By limiting the searches to English language articles for pragmatic reasons, it is also possible that published work exists in other languages but did not come to the attention of the reviewers.

Only 2 studies reported response rates; one was low at $10.5 \%$ [13] and raises the prospect of responder bias. Another study reported a response rate of more than $90 \%$ [11]; however, the survey was distributed to attendees at a dermatology conference for GPs and may not be representative of the GP population. One study reviewed data from a skin cancer audit database used by GPs, and doctors selfselect to participate in this tool [7]. It is therefore difficult to assess how well these data reflect the use of dermoscopy among GPs generally.

The focus of the review was narrowed to looking at GPs within a primary care, generalist setting. This inevitably excluded articles reporting on GPs working in specialist skin cancer care or in a hospital setting. Presumably some GPs working in specialist settings may also work in a generalist setting at times and would bring their dermoscopy skills with them to this role. However, most patients in health care systems where GPs play a "gate-keeping” role for secondary care will attend a GP without these enhanced skills. Therefore, it was important for our team to understand how a GP working in a generalist setting uses dermoscopy.

\section{Implications}

Several important gaps in the literature were uncovered in this review. From the authors' perspective, the absence of published data from the UK or Ireland means that there is currently no clear understanding of how many GPs use dermoscopy in these jurisdictions. All health care systems vary, and if an expansion of the use of dermoscopy in primary care were to be planned, having some baseline data on the use of dermoscopy would be highly desirable.
All of the studies included in this review were observational, and there have been no long-term or follow-up studies that have looked at how the use of dermoscopy has changed in general practice over time. While this is partly due to the relatively novel status of dermoscopy as a tool for GPs, it means that trends in dermoscopy use in primary care are currently unknown or unpublished. Furthermore, because questionnaire studies have not used identical questions, comparison of dermoscopy use between different health care systems and countries, such as has been done in a survey of dermatologists [17], is not yet possible for GPs.

This review highlights a concerning absence of qualitative research into GPs' use of dermoscopy. While questionnaires have attempted to highlight GPs' perceptions of the advantages of dermoscopy and the barriers to its use, they inevitably do so at a superficial level. Questions such as why most GPs do not use dermoscopy and whether specific perceptions or barriers are preventing them from doing so have not been addressed. Understanding these factors is crucial to understanding how dermoscopy can be translated from a new tool for lesion recognition into a standard technique for the assessment of skin lesions in primary care.

Notably, one of the perceived barriers to the use of dermoscopy in primary care highlighted by this review is the need for training. One of the papers included in this review reported on how GPs train in dermoscopy, and found that only a minority of dermoscopy users had undertaken training [13]. It is unsurprising that many GPs are unwilling to incorporate dermoscopy into their clinical practice without undertaking training, and understanding how to train GPs in dermoscopy will be important in expanding use of dermoscopy among the GP workforce.

\section{Conclusions}

This scoping literature review found research from several countries that demonstrates that dermoscopy is used by a minority, often a small minority, of GPs. Commonly perceived barriers to the use of dermoscopy include equipment costs and the time and training required to use it. No published qualitative research has explored these perceptions, and further research in this area could help to lay foundations for more widespread uptake of dermoscopy among GPs. In particular, understanding how GPs train in dermoscopy will be important in improving patient access to dermoscopy in primary care.

\section{References}

1. Herschorn A. Dermoscopy for melanoma detection in family practice. Can Fam Physician. 2012;58(7):740-745 [in English, French]. 
2. Pham MT, Rajić A, Greig JD, Sargeant JM, Papadopoulos A, Mcewen SA. A scoping review of scoping reviews: advancing the approach and enhancing the consistency. Res Synth Methods. 2014;5(4):371-385

3. Arksey H, O'Malley L. Scoping studies: towards a methodological framework. Int J Soc Res Methodol. 2005;8(1):19-32.

4. Levac D, Colquhoun H, O'Brien KK. Scoping studies: advancing the methodology. Implement Sci. 2010;5:69. PMID: 20854677.

5. Moher D, Liberati A, Tetzlaff J, et al. Preferred Reporting Items for Systematic Reviews and Meta-Analyses: the PRISMA statement. PLoS Med. 2009;6(7):e1000097.

6. Peters MD, Godfrey CM, Khalil H, McInerney P, Parker D, Soares CB. Guidance for conducting systematic scoping reviews. Int J Evid Based Healthc. 2015;13(3):141-146.

7. Rosendahl C, Williams G, Eley D, et al. The impact of subspecialization and dermatoscopy use on accuracy of melanoma diagnosis among primary care doctors in Australia. J Am Acad Dermatol. 2012;67(5):846-852.

8. World Bank Country and Lending Groups. The World Bank. Available at: https://datahelpdesk.worldbank.org/knowledgebase/ articles/906519\#High_income. Accessed September 12, 2018.

9. International Monetary Fund. 2018. World Economic Outlook: Cyclical Upswing, Structural Change. Washington, DC, April. Available at: http://www.imf.org/en/Publications/WEO/ Issues/2018/03/20/world-economic-outlook-april-2018. Accessed September 12, 2018.

10. United Nations Development Programme. Human Development Report 2016: Human Development for Everyone. 2016.
Available at: http://hdr.undp.org/sites/default/files/2016_human_ development_report.pdf. Accessed September 12, 2018.

11. Chamberlain AJ, Kelly JW. Use of dermoscopy in Australia. Med J Aust. 2007;187(4):252-253.

12. Secker LJ, Buis PAJ, Bergman W, Kukutsch NA. Effect of a dermoscopy training course on the accuracy of primary care physicians in diagnosing pigmented lesions. Acta Derm Venereol. 2017;97(2):263-265.

13. Chappuis P, Duru G, Marchal O, Girier P, Dalle S, Thomas L. Dermoscopy, a useful tool for general practitioners in melanoma screening: a nationwide survey. Br J Dermatol. 2016;175(4):744750 .

14. Ahmadi K, Prickaerts E, Smeets JGE, Joosten V, Kelleners-Smeets NWJ, Dinant GJ. Current approach of skin lesions suspected of malignancy in general practice in the Netherlands: a quantitative overview. J Eur Acad Dermatology Venereol. 2018;32(2):236241. Epub 2017 Aug 16.

15. Morris JB, Alfonso SV, Hernandez N, Fernández MI. Dermascope use by osteopathic primary care physicians. J Am Osteopath Assoc. 2017;117(3):158-164.

16. Morris JB, Alfonso SV, Hernandez N, Fernández MI. Examining the factors associated with past and present dermoscopy use among family physicians. Dermatol Pract Concept. 2017;7(4):6370.

17. Forsea AM, Tschandl P, Zalaudek I, et al. The impact of dermoscopy on melanoma detection in the practice of dermatologists in Europe: results of a pan-European survey. J Eur Acad Dermatol Venereol. 2017;31(7):1148-1156. 